\title{
Implications de données récentes sur les Lingules actuelles dans les interprétations paléoécologiques
}

CHRISTIAN C. EMIG

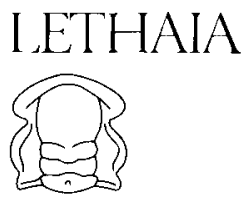

Emig, Christian C. 198107 01: Implications de données récentes sur les Lingules actuelles dans les interprétations paléoécologiques. IImplications of new data on Recent lingulid brachiopods in paleoccological interpretations.] Lethaia, Vol. 14, pp. 151-156. Oslo ISSN 0024-1164.

Some traditional concepts on lingulid paleoecology are reviewed and discussed in regard to new data on the ecology of Recent lingulid brachiopods. Fossilization in life-position occurs under several ecological conditions, e.g. dessication or emersion of the substratum, prolonged increase in salinity, perhaps very rapid sedimentation, extreme changes in temperature, and fossilization of flat-lying shells occurs under prolonged reduction in salinity, storms, natural death. Catastrophic environmental changes cause the fossilization of lingulids. Such events may affect one or more ecological features which may have a direct or indirect effect on the lingulid biology. At this time, the biotype does not correspond to the habitual one, and the survival and behavior of the lingulids depend on the severity of the environmental changes and on the tolerance of the animals to the latter.

Christian C. Emig, Station Marine d'Endoume (Laboratoire Associé au C.N.R.S. $n^{\circ} 41$ ), rue de la Batterie des Lions, 13007 Marseille, France; 24 juillet 1980.

Il est actuellement admis que les formes récentes de Lingulides sont caractérisées par une absence de changements évolutifs significatifs depuis l'Ordovicien (environ 500 millions d'années) pour le genre Lingula et depuis le début du Cénozoïque (environ 50 millions d'années) pour le genre Glottidia, ce qui devrait permettre d'étendre jusqu'à ces périodes les observations sur les espèces actuelles de Lingules (Craig 1952; Williams 1957; Paine 1963, 1970; Worcester 1969; Thayer \& Steele-Petrovic 1975; Emig et al. 1978; Plaziat et al. 1978; Emig 1980); ces auteurs ont dénoncé ou fourni des résultats remettant en cause des concepts traditionnellement véhiculés sur les Lingules, aussi rappellerons-nous ici brièvement quelques facteurs régissant la répartition des Lingules actuelles:

Les Lingules sont euryhalines (42-18\%o, parfois jusqu'à $15 \%$ o), bien que généralement infeodées à des milieux à salinités normales. La limite inférieure pour la température se situe entre 18 et $12^{\circ} \mathrm{C}$. Le substrat le plus favorable, voire préférentiel, est un sédiment sableux infralittoral (Fig. 1), pouvant s'étendre vers l'étage bathyal: sables fins se situant dans l'intervalle de 200 à $60 \mu \mathrm{m}$ pour le diamètre des particules. Il semble que la mise en saltation de particules dépassant environ $200 \mu \mathrm{m}$ sous l'effet de l'hydrodynamisme pourrait être un facteur limitant dans les zones en eaux peu profondes; de même que les particules fines (inférieures à $60 \mu \mathrm{m}$ ) provo- quent le colmatage de la cavité palléale. La tolérance des Lingules vis-à-vis de tous ces facteurs, auxquels il faut ajouter l'oxygénation du milieu, la nourriture disponible, la prédation, la faune environnante, est probablement bien moins grande que l'ont affirmé divers auteurs, tant actualistes que paléontologues.

Ces résultats sur les Lingules actuelles peuvent être appliqués à la paléoécologie de ce groupe zoologique pour mieux définir les conditions qui ont amené à leur fossilisation. C'est ce que nous nous proposons de faire, en discutant ensuite quelques exemples de faciès à Lingules fossiles particulièrement bien décrits dans des travaux précédents par d'autres auteurs.

\section{Terriers}

La longueur des terriers des Lingules, en position verticale (Fig. 2), est fonction de la nature du sédiment: dans les sables fins, homogènes, cette longueur peut atteindre une quarantaine de centimètres pour des individus, dont la coquille mesure de 3,5 à $4 \mathrm{~cm}$ (soit environ 10 fois la longueur de la coquille). Ceci est aussi l'extension maximale que peut atteindre le pédoncule de la Lingule. Ainsi, cette observation permet d'apprécier l'importance du tassement qui a affecté une strate sédimentaire dans laquelle se trouve des terriers fossiles. A titre d'exemple, 


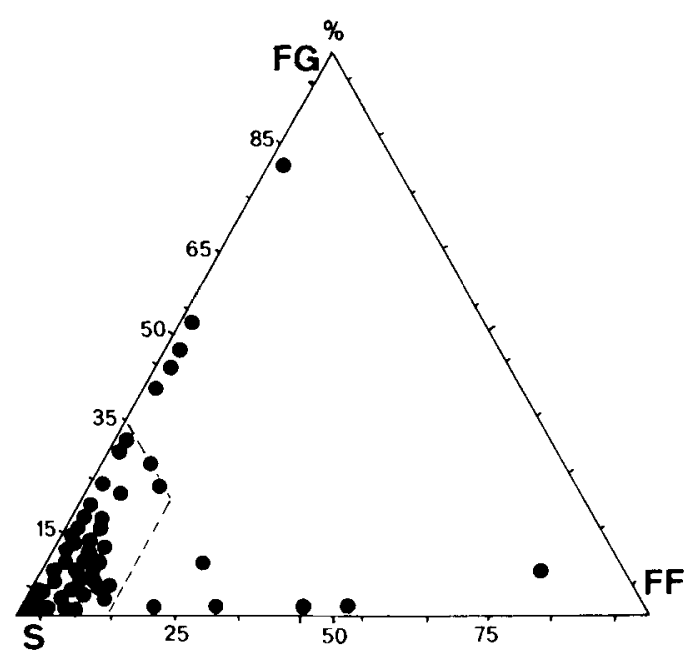

Fig. 1. Diagramme triangulaire des sédiments à Lingules actuelles, d'après les résultats publiés par Emig et al. (1978) et Emig (1980): $F G=$ fraction grossière supérieure à $2.5 \mathrm{~mm}$; $\mathrm{S}=$ sables de $2,5 \mathrm{~mm}$ à $40 \mu \mathrm{m} ; \mathrm{FF}=$ fraction fine inférieure à 40 $\mu \mathrm{m}$.

Equilateral triangular plots of the sediments occupied by recent Lingula, from data of Emig et al. (1978) and Emig (1980). $F G=$ particles greater than $2.5 \mathrm{~mm} ; S=$ sand from $2.5 \mathrm{~mm} t 040 \mathrm{um} ; \mathrm{FF}=$ le'ss than sand.

nous reprendrons le schéma d'un terrier fossile dans des grès argileux du Trias des Vosges (Fig. 2B): pour une coquille fossile d'environ un centimètre, le terrier devrait mesurer une dizaine de centimètres dans ces grès; or, il n'est que de 5 à $6 \mathrm{~cm}$ (soit environ 3,5 à $4 \mathrm{~cm}$ pour la partie supérieure, dans laquelle se déplace la coquille, et environ 2 à $2,5 \mathrm{~cm}$ pour la partie occupée par le seul pédoncule), les deux parties du terrier représentant respectivement $2 / 3$ et $1 / 3$ comme dans les terriers actuels (Fig. 2A). Le tassement du sédiment fût donc, pour le cas évoqué, de l'ordre du tiers à la moitié de son épaisseur originelle.

Par contre, quand la fraction très grossière augmente dans la profondeur du sédiment, comme par exemple dans la baie de Kaneohe (Hawaii) sous forme de débris coralliens, abondants sous une vingtaine de centimètres de sédiment, la longueur du terrier diminue pour n'atteindre que cette vingtaine de centimètres, même pour des coquilles dépassant $2 \mathrm{~cm}$ de long. Le pédoncule se glisse alors entre ces débris, qui accroissent la force d'ancrage du pédoncule, et l'accumulation de particules autour du renflement basal du pédoncule devient moins im-

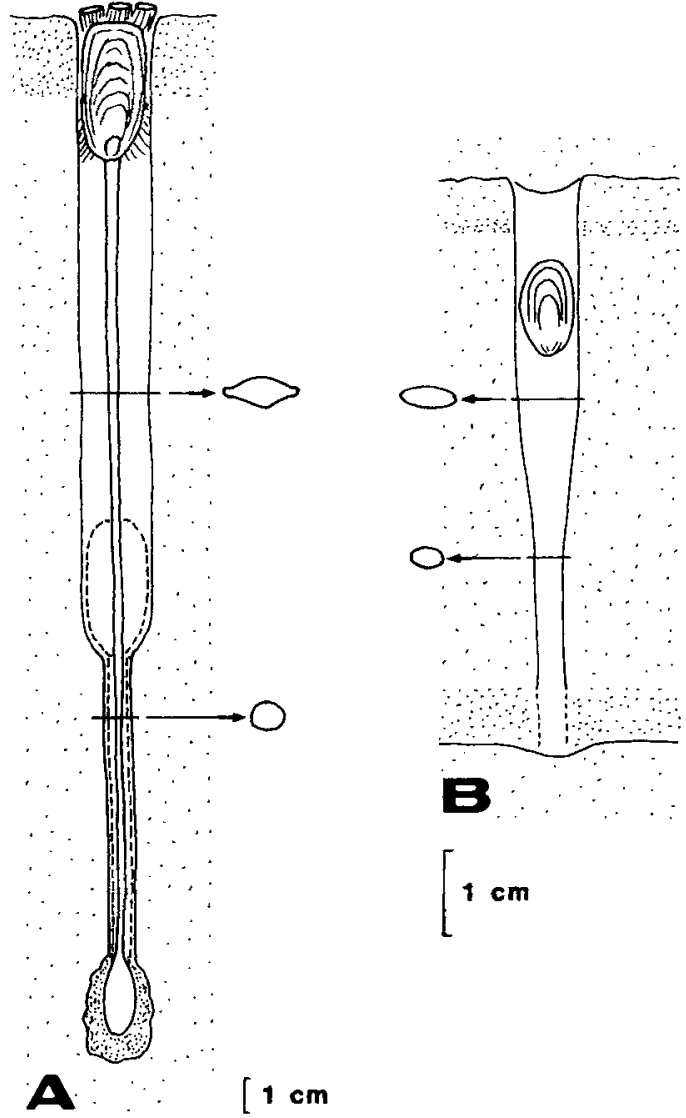

Fig. 2. $\square$ A. Schéma d'un terrier de Lingula révei (espèce actuelle), qui est représentée en position haute (position normale, dite en position de vie), et, en trait pointillé au fond du terrier. $\square$ B. Schéma d'une Lingule ('Lingula' tenuissima) in situ dans son terrier, dans le grès argileux du Trias des Vosges (d'après Emig et al. 1978).

$\square A$. Diagram of a burrow of Lingula reevei (recent species), in life-position (dotted line shows the animal retracted downward in its burrow). $\square$ B. Diagram of a lingulid ('Lingula' tenuissima) in its burrow in clay sandstone of the Trias of Vosges Mountains (from Emig et al. 1978).

portante que dans le cas précédent. Ces observations pourraient s'appliquer aux sables à Lingula à la base desquels existe une couche de 'shellrocks', décrits par Meyer (1871). Peut-être aussi aux descriptions faites par Newall (1970), les Lingules utilisant les perforations colmatées dans des coraux pour y faire leurs terriers, sans qu'il faille invoquer une possible relation symbiotique.

Il faut signaler ici que les mécanismes originaux de pénétration et de changements de 
terrier, décrit par Thayer \& Steele-Petrovic (1975) chez Glottidia pyramidata, ne semblent pas, selon Emig (1980), pouvoir être étendus au genre Lingula.

\section{Fossilisation}

Les Lingules fossiles ont été décrites soit en position de vie (rarement), soit sous forme de valves éparses, pouvant atteindre des accumulations très importantes. Grâce à des observations sur la biologie des Lingules actuelles (Emig 1980), une analyse des conditions de fossilisation est devenue possible.

\section{Fossilisation en position de vie}

La mort des Lingules en position de vie peut être consécutive aux modifications du milieu suivantes: - augmentation de la salinité du milieu, au-delà de 40 à $50 \%$; - émersion du substrat. Des expériences sur Lingula reevei ont démontré que les animaux ne résistent que pendant quelques jours à ces conditions défavorables, puis la mort survient, les Lingules restant dans leur terrier, généralement quelques centimètres sous l'ouverture du terrier. Cette fossilisation, quand elle se produit, est donc la conséquence de modifications brutales du milieu naturel, dans des zones littorales ou lagunaires, ou devenues telles sous l'effet de l'abaissement du niveau de la mer ou de l'émersion des fonds.

Gall (in Emig et al. 1978) a décrit la fossilisation d'une population de Lingules, 'Lingula' tenuissima Bronn, dans des grès du Trias des Vosges par suite d'une émersion des fonds, la zone étant littorale, soumise à une salinité probablement fluctuante et la couche d'eau ne devant pas excéder quelques décimètres. Ce milieu semble proche des biotopes infralittoraux deltaiques actuels de Nouvelle-Calédonie (dans lesquels une population de Lingula est en cours d'étude). Quelques remarques de portée générale sont à formuler: - d'abord, pour l'apport d'oxygène, les Lingules sont tributaires de la seule masse d'eau (ce sont des suspensivores) et non liées au sédiment dans lequel la couche oxydée peut ne pas dépasser $2 \mathrm{~cm}$ (Fig. 2A), sans que l'on puisse pour autant considérer le milieu comme peu oxygéné; - ensuite, comme le remarque aussi Ferguson (1963), la pyritisation des terriers ou des valves est liée à la décomposition des parties molles de l'animal, et non aux condi- tions anoxiques du milieu; - enfin, il se confirme, au vu de travaux récents (Emig et al. 1978; Emig \& LeLoeuff 1978; Kenchington \& Hammond 1979; Emig 1980), que les Lingules vivent dans des milieux marins à salinité normale. D'après des observations en Nouvelle-Calédonie (J. Picard, communication personnelle), la présence de Lingules en milieu deltaïque ne signifie nullement que le biotope, où ces animaux sont localisés, est soumis à des salinités réduites ou fluctuantes. Cherns (1979) a signalé deux espèces fossiles, Lingula lata et $L$. lewisii, en position de vie probablement à des profondeurs atteignant $1500 \mathrm{~m}$, en eaux marines franches.

Le comportement des Lingules devant un rapide accroissement de la sédimentation, notamment en particules grossières ou fines, reste inconnu. Des expériences ont montré que la Lingule peut suivre une sédimentation sableuse modérée et se maintenir à la surface du substrat, même si l'autotomie du pédoncule, qui régénère, s'avère nécessaire (Emig 1980). De même, nous ignorons la réaction des Lingules, si ce n'est une mortalité massive, durant un phénomène d'aux rouges, provoquant des conditions anaérobies (Simon \& Dauer 1977).

\section{Fossilisation des valves}

La déssalure du milieu (sous environ 15\% ) entraine, en quelques jours, la mort d'une population de Lingules. Celles-ci sortent alors de leur terrier et la putréfaction des chairs provoque la séparation des valves, qui jonchent le fond. La mort après extrusion des animaux hors du terrier peut aussi être la conséquence du démantèlement du substrat sous l'action d'une tempête ou d'un cyclone: de tels phénomènes ont été observés aux Indes par Ramamoorthi et al. (1973), aux Philippines où Fenton (1966) a signalé des amas de valves de Lingules atteignant 30 pouces de haut, en Australie. D'après Johnston \& Hirschfeld (1920), Worcester (1969), la mort 'naturelle' des Lingula conduirait à l'extrusion de l'animal hors de son terrier, devenant alors une proie facile pour des prédateurs.

Ces valves peuvent, ensuite, être entraînées par des courants et s'accumuler en plus ou moins grand nombre dans des cuvettes à décantation, généralement à sédiment vaseux, où ces valves peuvent fossiliser plus ou moins loin de leur biotope d'origine. La flottation des valves sous l'effect de la putréfaction des chairs, évoquée par plusieurs auteurs, apparaît en fait n'être 
qu'un phénomène secondaire et occasionnel (Emig 1980). En milieu récifal, les valves sont très rapidement détruites sous l'effet d'actions mécaniques et biochimiques (Worcester 1969: Emig 1980).

\section{Interprétations paléoécologiques de quelques gisements à Lingules}

Nous discuterons les résultats des travaux de Ferguson $(1962,1963)$ et d'Alexandrovicz \& Jarosz (1971) au vu des faits établis ci-dessus. Le choix s'est limité à ces deux cas, car ce sont les seuls auteurs ayant décrit dans le détail un gisement à Lingules fossiles permettant une discussion sur les conditions ayant entrainé la fossilisation d'une population de Lingules. Dans ces deux exemples, la fossilisation des Lingules est consécutive à une transgression marine sous des conditions fluctuantes du milieu à la limite de tolérance de ces animaux.

D'après Ferguson $(1962,1963)$, les coquilles de Lingula squamiformis Phillips, découvertes en Ecosse dans le Carbonifère (Mississipien supérieur), mesuraient en général de 9 à $15 \mathrm{~mm}$ (de 1,25 à $25 \mathrm{~mm}$ ): $25 \%$ o de ces coquilles étaient en position de vie dans des terriers verticaux ou légèrement inclinées, les autres étant parallèles au litage (soit avec les deux valves d'un même individu proches l'une de l'autre, attestant l'absence de courants pour les déplacer, soit parsemées sous l'action des seuls courants de marées). Par rapport à l'épaisseur de la strate, les animaux en position de vie se situaient principalement entre 0 et $25 \mathrm{~cm}$ et 75 et $150 \mathrm{~cm}$ (Fig. 3): dans le premier cas, la mort serait intervenue en eau peu profonde par suite de changements brutaux des conditions de milieu. En reprenant certains des éléments proposés par Ferguson (1963), nous pouvons expliquer cette mortalité par une salinité très fluctuante (notamment sous l'effet d'averses tropicales) et par une sédimentation très fine importante. Des conditions similaires semblent être responsables de la fossilisation de la population de $L$. squamiformis, décrite par Craig $(1952,1954)$ dans une strate-sédimentaire composée d'un horizon inférieur de sables fins et d'un horizon supérieur de marne micacées, strate dans laquelle $70 \%$ des Lingules fossiles sont en position de vie. Rappelons que, si la fraction fine (inférieure à environ $60 \mu \mathrm{m}$ ) devient trop importante, elle entraîne la mort des ani-

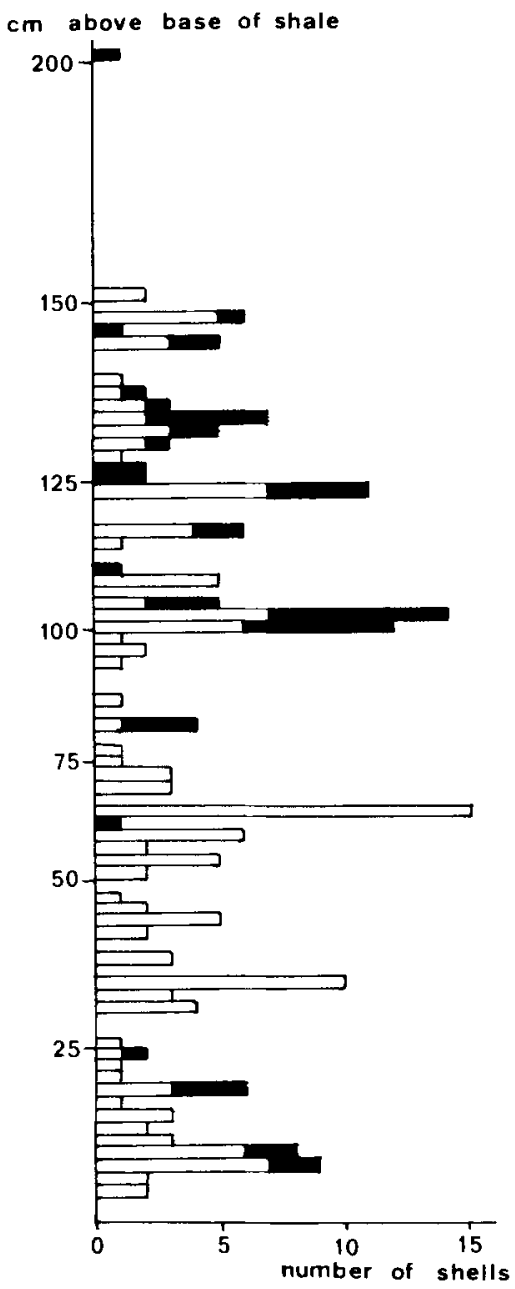

Fig. 3. Distribution de Lingula squamiformis dans les $200 \mathrm{~cm}$ inférieurs de la strate sous le Second Abden Limestone (Carbonifère inférieur): en diagramme blanc les valves couchées, en noir les lingules en position de vie (d'après Ferguson 1963).

Relative distribution of flat-lying specimens (represented in white) and inclined specimens (represented in black) of Lingula squamiformis in the lower $200 \mathrm{~cm}$ of the $280 \mathrm{~cm}$ of shale below the Second Abden Limestone (Lower Carboniferous). From Ferguson (1963).

maux par asphyxie par colmatage de la cavité palléale. Pour les Lingules fossilisées entre 75 et $150 \mathrm{~cm}$, Ferguson (1963) propose comme explication une mortalité liée à une diminution de la température des eaux par suite de l'enfoncement des fonds à une profondeur, variant de 45 à $90 \mathrm{~m}$ environ, phénomème accompagné par une chute de la nourriture disponible. Nous ignorons les 

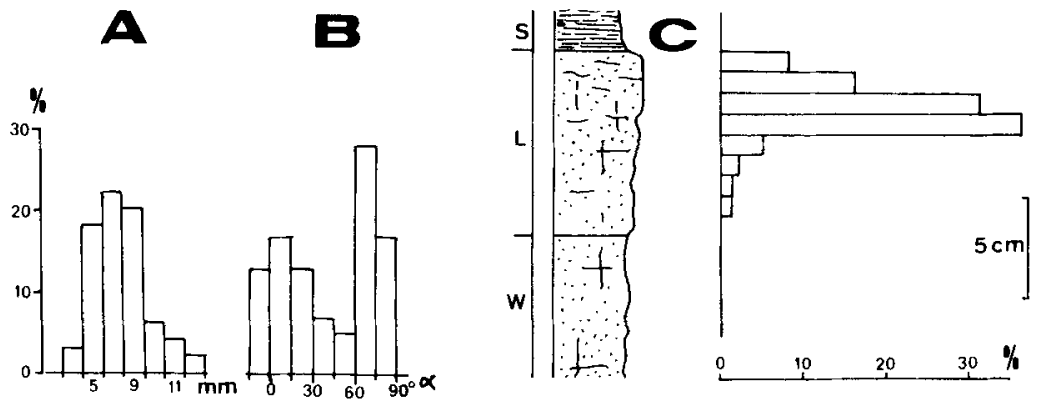

Fig. 4. Lingula credneri dans les grès du Zechstein (Permien supérieur). D’après Alexandrowicz \& Jarosz (1971). $\square$ A Distribution des coquilles en fonction de leur longueur. $\square$ B. Inclinaison des coquilles par rapport à la surface de la strate. $\square \mathrm{C}$. Histogramme de dispersion des coquilles de Lingules dans la strate de grès (S: copper shales; L: Lingula sandstone; W: white liny sandstone)

Lingula credneri in Zechstein sandstones (Upper Permian). From Alexandrowicz \& Jarosz (1971). $\square$ A. Diagram of shell length distribution. $\square B$. Angle of shell length inclination. $\square$ C. Frequency of occurrence of Lingula in the layer of Lingula sandstone (S: copper shales; $L$ : Lingula sandstone; $W$ : white liny sandstone).

réactions des Lingules à l'abaissement de température, mais cette hypothèse de Ferguson (1963) est à mettre en parallèle avec les résultats de Pickerill ( 1973), qui décrit Lingulasma tenuigranulata en position de vie dans une paléo-communauté benthique profonde du plateau continental (Ordovicien, Pays de Galles), et, pour cette espèce, la mortalité pourrait être liée à une baisse de température. Il conviendrait donc de vérifier expérimentalement la température léthale et le comportement des Lingules lors de la mort (maintien dans le terrier ou sortie hors de celui-ci).

Alexandrovicz \& Jarosz (1971) ont découvert Lingula credneri Geinitz dans des sables fins, en Pologne dans le Zechstein inférieur (Permien supérieur), mis en place en eau chaude peu profonde (milieu subtropical): environ 50\% des Lingules étaient en position de vie dans la partie supérieure de la couche $\mathrm{L}$ (Fig. 4B, C), alors que les valves couchées étaient surtout localisées un peu au-dessous, les dimensions des valves étant similaires dans les deux cas (Fig. 4A). D'après ces auteurs, le biotope aurait été soumis à un changement brutal des conditions de milieu ou à une rapide sédimentation, tandis que l'absence d'autres fossiles peut être expliquée par une réduction anormale de la salinité. L'interprétation que nous proposons invoque en premier lieu un abaissement de la salinité du milieu (environ $15 \%$ ) qui aurait alors provoqué la mort d'une partie de la population, les Lingules sortant du terrier et leurs valves jonchant la surface du sédiment; en second lieu, une rapide sédimenta- tion (Fig. 4C, couche $S$ ) que les Lingules encore vivantes auraient essayé de compenser, d'où leur position au-dessus des valves couchées, sans toutefois y parvenir en raison de leur état physiologique déficient. Ceci peut aussi expliquer l'absence de terrier nettement marqué. L'interprétation de cet exemple est aussi à mettre en parallèle avec celles concernant Ferguson (1963) et Craig (1952), discutées ci-dessus.

\section{Conclusions}

Des données sur l'écologie et la biologie des Lingules actuelles ont permis d'analyser les conditions de fossilisation et ainsi de faciliter l'interprétation de gisements à Lingules fossiles.

Pour conclure, il nous paraît souhaitable d'attirer l'attention sur les deux points suivants:

Si les formes actuelles de Lingules vivent dans des sables fins (Fig. 1), il conviendra pour le géologue d'étudier attentivement la granulométrie des couches dans lesquelles se situent les Lingules fossiles et d'établir sans hâte les causes de leur mort, notamment quand ces animaux sont découverts dans d'anciennes vasières, que certains auteurs interprètent systématiquement comme le biotope habituel des Lingules du Paléozoïque, hypothèse qui ne repose sur aucun fondement.

$\mathrm{La}$ fossilisation des Lingules intervient toujours quand le milieu subit des modifications brutales et rapides, c'est-à-dire pendant l'intervalle de vie des Lingules (5-7 ans, voire 12 ans 
pour le genre Lingula; 1-2 ans pour certaines espèces de Glottidia). Ces modifications peuvent affecter un ou plusieurs facteurs (salinité, température, sédiment ...) et intervenir soit directement sur la biologie des Lingules, soit indirectement (prédation, nourriture disponsible, ...). Dès lors, le biotope ne correspond plus à celui dans lequel vivaient habituellement les Lingules. Leur survie et leur comportement ne sont plus que fonction de la gravité et des conséquences de ces modifications du milieu et de la tolérance des Lingules vis-à-vis de celles-ci. Ces faits obligent le géologue à manifester une plus grande prudence quant à l'emploi des Lingules comme indicateurs de milieu et à mener une discussion paléoécologique précise du faciès à Lingules en utilisant au mieux les connaissances sur les Lingules récentes.

\section{Références}

Alexandrowicz, S. W. \& Jarosz, J. 1971: Palaeoecology of the Zechstein Lingula sandstones from Lubin (West Poland). Bull. Acad. Polon. Sci., Sir. Sci. Terre, 29 (3), 183-191.

Cherns, Lesley 1979: The environmental significance of Lingula in the Ludlow series of the Welsh Borderland and Wales. Lethaia 12, 35-46.

Craig, G. Y. 1952: A comparative study of the ecology and palaeoecology of Lingula. Trans, Edin. Geol. Soc. 15, $110-120$.

Craig, G. Y. 1954: The palaeoecology of the Top Hosie Shale (Lower Carboniferous) at a locality near Kilsyth. Q. J. Geol. Soc. Lond. I 10, 103-119.

Emig, C. C. 1980: Observations sur l'ecologie de Lingula reevei Davidson (Brachiopoda: Inarticulata). J. exp. Mar. Biol. Eiol. (sous presse).

Emig, C. C. \& Le Loeuff, P. 1978: Description de Lingula parva Smith (Brachiopoda, Inarticulata), récoltée en Côte d'Ivoire, avec quelques remarques sur l'écologie de l'espèce. Tothys 8 (3), $271-274$.

Emig, C. C., Gall, J. C., Pajaud, D. \& Plaziat, J. C. 1978: Réflexions critiques sur l'écologie et la systématique des Lingules actuelles et fossiles. Géobios [1(5), 573-609.
Fenton, C. L. 1966: Tales Told by Fossils. 182 pp. Doubleday, Garden City, New York.

Ferguson, L. 1962: The palaeoecology of a Lower Carboniferous marine transgression. J. Paleontol. 36(5), 1090-1107.

Ferguson, L. 1963: The palaeoecology of Lingula squamiformis Phillips during a Scottish Mississippian marine transgression. J. Paleontol. 37(3), 669-681.

Johnston, T. H. \& Hirschfeld, O. S. 1920: The Lingulidae of the Queensland Coast, Proc. R. Soc. Qd 31, 46-84.

Kenchington, R. A. \& Hammond, L. S. 1978: Population structure, growth and distribution of Lingula anatina (Brachiopoda) in Queensland, Australia. J. Zool. Lond. 184, 63-81.

Meyer, C. J. A. 1871: On Lower Tertiary deposits recently exposed at Portsmouth. Q. J. Geol. Soc. Lond. 27, 74-89.

Newall, G. 1970: A symbiotic relationship between Lingula and the coral Heliolithe's in the Silurian. In Crimes, T. P. \& Harper, J. C. (eds.): Trace Fossils, 335-344, Seel House Press, Liverpool.

Paine, R. T. 1963: Ecology of the Brachiopod Glottidia pyramidata. Ecol. Monogr. 33(3), 187-213.

Paine, R. T. 1970: The sediment occupied by recent lingulid brachiopods and some paleoecological implications. Palaeogeogr. Palaeoclim. Palaeoecol. 7, 21-31.

Pickerill, R. K. 1973: Lingulasma t'nuigranulata Palaeoecology of a large Ordovician Linguloid that lived within a strophomenid-trilobite community. Palaevgeogr. Palaeoclim. Palaeoccol. 13, 143-156.

Plaziat, J. C., Pajaud, D., Emig, C. C. \& Gall, J. C. 1978; Environnements et distribution bathymétrique des Lingules actuelles: consequences pour les interpretations paléogéographiques. Bull. Soc' geo. France 20(3), 309-314.

Ramamoorthi, K., Ventkataramanujam, K. \& Srikrishnadhas, B. 1973: Mass mortality of Lingula anatina (Lam.) (Brachiopoda) in Porto-Novo waters, S. India. Curr. Sci. 42(8), 285-286.

Simon, J. L. \& Dauer, D. M. 1977: Reestablishment of a benthic community following natural defaunation. In Coull, B. C. (ed.): Ecology of marine benthos. Marine Sciences 6 . 139-154.

Thayer, C. W. \& Steele-Petrović, H. Miriam 1975: Burrowing in the lingulid brachiopod Glottidia pyramidata: it ecologic and paleoecologic significance. Lethaia 8, 209-221.

Williams, J. S. 1957: Paleoecology of the Mississippian of the Upper Mississippi valley region. In Ladd, H. S. (ed.): Treatise on marine ecology and paleoecology. Geol, Soc. Am. Wash. Mem. 67(2), 279-324.

[Worcester, W. 1969: On Lingula reevei. Unpublished M.S. Thesis, Univ. Hawaii, Honolulu. 49 pp.] 